\title{
Philosophiques
}

\section{Albert Lautman, ou la dialectique dans les mathématiques}

\section{Brendan Larvor}

Volume 37, numéro 1, printemps 2010

Albert Lautman, philosophe des mathématiques

URI : https://id.erudit.org/iderudit/039713ar

DOI : https://doi.org/10.7202/039713ar

Aller au sommaire du numéro

Éditeur(s)

Société de philosophie du Québec

ISSN

0316-2923 (imprimé)

1492-1391 (numérique)

Découvrir la revue

Citer cet article

Larvor, B. (2010). Albert Lautman, ou la dialectique dans les mathématiques. Philosophiques, 37(1), 75-94. https://doi.org/10.7202/039713ar

\section{Résumé de l'article}

Dans cet article, j'explore dans un premier temps la conception que se fait Lautman de la dialectique en examinant ses références à Platon et Heidegger. Je compare ensuite les structures dialectiques identifiées par Lautman dans les mathématiques contemporaines avec celles qui émergent de ses sources philosophiques. Enfin, je soutiens que les structures qu'il a découvertes dans les mathématiques sont plus riches que le suggère son modèle platonicien, et que la distinction « ontologique » de Heidegger est moins utile que semblait le penser Lautman. 


\title{
Albert Lautman, ou la dialectique dans les mathématiques
}

\author{
BRENDAN LARVOR \\ University of Hertfordshire \\ b.p.larvor@herts.ac.uk
}

\begin{abstract}
RÉSUMÉ. - Dans cet article, j'explore dans un premier temps la conception que se fait Lautman de la dialectique en examinant ses références à Platon et Heidegger. Je compare ensuite les structures dialectiques identifiées par Lautman dans les mathématiques contemporaines avec celles qui émergent de ses sources philosophiques. Enfin, je soutiens que les structures qu'il a découvertes dans les mathématiques sont plus riches que le suggère son modèle platonicien, et que la distinction «ontologique» de Heidegger est moins utile que semblait le penser Lautman.
\end{abstract}

\begin{abstract}
In this paper, I first explore Lautman's conception of dialectics by a consideration of his references to Plato and Heidegger. I then compare the dialectical structures that he found in contemporary mathematics with the model that emerges from his philosophical sources. Finally, I argue that the structures that he discovered in mathematics are richer than his Platonist model suggests, and that Heidegger's "ontological" distinction is less useful than Lautman seemed to believe.
\end{abstract}

[1] Albert Lautman (1908-1944) est un rare exemple d'un philosophe du vingtième siècle dont l'intérêt pour les mathématiques contemporaines s'étend au-delà des domaines «fondationnels » de la logique mathématique et de la théorie des ensembles. Il affirme que ce qui constituait à son époque les nouvelles mathématiques de la topologie, de l'algèbre abstraite, de la théorie du corps de classes et de la théorie analytique des nombres a une importance philosophique qui les distingue des mathématiques des époques antérieures. Ces nouveaux domaines des mathématiques révèlent en particulier des structures dialectiques sous-jacentes inconnues dans les mathématiques du passé. Dans une série de courts articles et dans deux thèses plus longues (Essai sur l'unité des sciences mathématiques dans leur développement actuel et Essai sur les notions de structure et d'existence en mathématiques $^{1}$ ), Lautman soutient ce point de vue à partir d'une perspective philosophique qui trouve son origine dans certains des derniers dialogues de Platon. Toutefois, la conception platonicienne de la relation entre les Idées dialectiques et la matière dans laquelle elles sont réalisées ne satisfaisait pas Lautman. Dans l'un de ses derniers articles, "Nouvelles recherches sur la

1. Lautman, 2006. Les numéros de page référeront dans la suite du présent texte à l'édition complète des œuvres de Lautman parues chez Vrin en 2006. 
structure dialectique des mathématiques $»^{2}$, Lautman supplémente son platonisme en recourant à la distinction "ontologique» de Heidegger entre la phénoménologie et la science ${ }^{3}$. Nous pouvons par conséquent considérer cet article comme l'expression la plus poussée qui soit disponible quant à la philosophie des mathématiques de Lautman.

Dans le présent article, je commencerai mon examen de la conception de la dialectique de Lautman en considérant les références de ce dernier à Platon et Heidegger. Je comparerai ensuite les structures dialectiques qu'il repère dans les mathématiques contemporaines avec le modèle qui se dégage des sources philosophiques auxquelles il se réfère. Je soutiendrai que les structures que Lautman découvre dans les mathématiques sont plus riches que ne le suggère son modèle platoniste, et que la distinction «ontologique» heideggerienne est moins utile qu'il ne semble le croire.

\section{Platon}

Lautman décrit à l'aide d'exemples approfondis les mathématiques modernes (c'est-à-dire les mathématiques du début du vingtième siècle) comme l'expression ou la réalisation d'oppositions conceptuelles fondamentales (telles que celles du continu et du discontinu, du global et du local, du fini et de l'infini, du symétrique et de l'antisymétrique) ${ }^{4}$. Il appelle ces termes opposés des «notions"; les «Idées» dialectiques envisagent des relations possibles entre des couples de notions dialectiques (pp. 242-243). Cette terminologie est une référence consciente à Platon. Lautman prend cependant soin de distinguer son recours à Platon du "platonisme » au sens où les philosophes des mathématiques utilisent ordinairement le terme. En philosophie des mathématiques, le "platonisme» réfère habituellement à la thèse selon laquelle les objets mathématiques existent indépendamment de la pensée et du discours des mathématiciens. [2] Lautman a soutenu qu'une telle conception était une mésinterprétation de Platon (p. 230); quoi qu'il en soit, cette sorte de "platonisme» n'est pas celle de Lautman".

Lautman ne cite jamais Platon directement, et il mentionne seulement trois textes platoniciens: le Philèbe, le Sophiste (deux fois) et le Timée (deux fois). Les spécialistes rangent ordinairement ces trois textes parmi les dialogues

2. Pp. 235-237. Publié pour la première fois en 1939 dans une collection dirigée par Jean Cavaillès et Raymond Aron.

3. Telle qu'exprimée par Heidegger dans sa conférence intitulée Von Wesen des Grundes. Lautman cite la traduction française donnée par Henry Corbin en 1938 (in Qu'est-ce que la métaphysique? Paris, Gallimard, 1938).

4. Cette liste est tirée des deux longs essais. Dans les « Nouvelles recherches », Lautman propose une liste légèrement différente de couples dialectiques: «le tout et la partie, les propriétés de situation et les propriétés intrinsèques, les domaines de base et les êtres définis sur ces domaines, les systèmes formels et leurs réalisations, etc. ", op. cit., p. 243.

5. «Dans le débat ouvert entre formalistes et intuitionnistes, [...] les mathématiciens ont pris l'habitude de désigner sommairement sous le nom de platonisme toute philosophie pour 
«tardifs» de Platon (bien que le Sophiste constitue la suite du Théétète et réfère implicitement au Parménide - deux dialogues de la "période moyenne »). L'important pour notre propos est que la «théorie des formes» de Platon soit largement absente de ses œuvres plus tardives. Les «Idées» dans les derniers dialogues ne sont pas des modèles pour les objets matériels. De la même façon, le platonisme mathématique de Lautman n'est pas une «théorie de la copie». Comme il le fait remarquer, nous pouvons concevoir la réalité matérielle comme de la matière inchoative façonnée de manière à constituer en quelque sorte des copies matérielles de "formes" non matérielles, mais ce modèle ne peut pas s'appliquer à la relation entre les théories mathématiques et les idées dialectiques qui (selon l'expression de Lautman) les «dominent» (p. 238).

\section{Le Sophiste}

Dans un court article datant de 1937 et intitulé «L'axiomatique et la méthode de division» (pp. 69-80), Lautman fait référence à la fois au Philèbe et au Sophiste:

Le passage des notions dites «élémentaires» aux notions abstraites ne se présente donc pas comme une subsomption du particulier sous le général mais comme la division ou l'analyse d'un «mixte» qui tend à dégager les notions simples auxquelles ce mixte participe. Ce n'est donc pas la logique aristotélicienne, celle des genres et des espèces qui intervient ici, mais la méthode platonicienne de division, telle que l'enseignent le Sophiste et le Philèbe pour laquelle l'unité de l'Être est une unité de composition et un point de départ vers la recherche des principes qui s'unissent dans les Idées (pp. 78-79).

Le Sophiste est composé d'une discussion entre un jeune homme, Théétète, et un étranger originaire d'Élée, qui «appartient au cercle des disciples de Parménide et de Zénon et [qui] est un véritable philosophe» (216A). La question initiale est de savoir si les mots «sophiste ", " homme politique » et «philosophe» désignent un, deux ou trois genres de choses, et quelle est, ou quelles sont, cette ou ces choses. L'étranger dont le nom n'est pas divulgué réclame un «interlocuteur complaisant et docile» (217D); Socrate propose le jeune Théétète. Platon donne ainsi la chance au philosophe anonyme d'exposer en long et en large sa position sans avoir à parer les attaques d'une interrogation socratique (cela est typique des dernières œuvres de Platon; dans le dialogue du même nom, Timée est seul à prendre la parole après les civilités d'usage). Après quoi Socrate disparaît [3] du texte, sans par conséquent nous offrir le luxe d'inférer le point de vue de Platon des phrases mises dans la bouche de Socrate.

laquelle l'existence d'un être mathématique est tenue pour assurée [...] c'est là une connaissance superficielle du platonisme...», p. 230. 
Le philosophe éléate procède par division, c'est-à-dire par distinctions successives. Il illustre cette technique par l'exemple de la "pêche à la ligne ". Il distingue d'abord les arts d'acquisition des arts de production; puis les arts d'acquisition sont divisés en arts d' «échange » et de "capture " ou de conquête; les arts de capture se divisent en compétition et en chasse; la chasse se divise selon la proie; les animaux nagent ou marchent; ceux qui nagent se divisent en oiseaux et en poissons; la pêche se divise en piégeage (à l'aide de filets, de pièges, etc.) et en "pêche frappeuse"; la pêche frappeuse se divise selon qu'elle procède de haut en bas à l'aide d'un trident ou de bas en haut à l'aide d'un hameçon. L'arbre de catégories obtenu au terme de ce processus constitue sa définition de "pêche à la ligne». Il applique ensuite la même technique au terme "sophiste", et cette discussion occupe le reste du dialogue. Le philosophe éléate développe plusieurs définitions du terme "sophiste»(231D-E), ce qui conduit à une discussion méthodologique incluant un débat sur la possibilité de dénombrer les non-êtres (238B). La discussion fait référence à elle-même, car Théodore présente l'Étranger éléate comme un philosophe, vraisemblablement en raison de sa technique logique $(253 \mathrm{C})^{6}$. Mais si la méthode de division se révèle n'être qu'un jeu verbal gratuit, alors peut-être est-il un sophiste. Ses choix de catégories de division semblent certes arbitraires. Par exemple, il aurait pu diviser la pêche selon qu'un appât est ou non utilisé, auquel cas la pêche au trident et la pêche au filet se seraient retrouvées dans une catégorie différente de la pêche à la ligne et du piégeage. Le jeune Théétète défère un peu trop facilement à l'autorité du philosophe, et certes plus facilement que Socrate ne l'aurait fait.

Quelle qu'ait été l'intention de Platon en montrant autant d'indulgence envers un philosophe éléate générique et anonyme, Lautman considère quant à lui la méthode de division comme une technique non problématique et ne fait aucune mention de celle qui lui est associée, la "méthode de collection ". Immédiatement avant le passage cité ci-dessus, Lautman énumère dans son texte une liste de "mixtes ", c'est-à-dire de notions mathématiques qui "participent " à deux catégories, à savoir: l'égalité arithmétique, seule relation d'équivalence telle que le nombre de classes d'équivalence est égal à la cardinalité du domaine de base ${ }^{7}$; l'idée de multiplication, qui «contient à la fois la formation de produits arithmétiques et l'action d'opérateurs sur un domaine d'éléments distincts de ces opérateurs» (p. 78); l'idée d'unité, qui "peut être considérée, soit du point de vue de l'élément unité d'un anneau de nombres, soit du point de vue de l'opérateur identique d'un domaine

6. Voir cependant l'argument de Trevaskis (1967) selon lequel la technique du philosophe ne se réduit pas à la méthode de division.

7. Texte exact de Lautman: «seule relation d'équivalence telle que le dénombrement des individus d'un ensemble se confonde avec le dénombrement des classes d'individus équivalents au sens de cette relation", in "L'axiomatique et la méthode de division", p. 78 (NDLT). 
d'opérateurs» (p. 78); la longueur d'un segment, qui « est liée à la grandeur qu'elle mesure mais [...] n'est » [en même temps] "qu'un nombre attaché par convention à cette grandeur» (p. 78); la valeur absolue dans l'algèbre classique, qui «enveloppe à la fois l'idée d'ordonnance et la construction de la fermeture d'un corps de nombres» (p. 78). Il poursuit en affirmant que certains de ces mixtes (l'égalité arithmétique; la multiplication; la valeur absolue) sont des exemples de la relation dialectique entre les propriétés intrinsèques et les propriétés relationnelles des objets mathématiques (pp. 78-79). Il suggère ensuite que «la distinction qui s'établit ainsi au sein d'une même notion entre les propriétés intrinsèques d'un être ou d'une notion et ses possibilités d'action nous semble s'apparenter à la distinction platonicienne du Même et de l'Autre qui se retrouvent dans l'unité de l'être " (p. 79). Pour Lautman, par conséquent, ces notions mathématiques (égalité, [4] multiplication, unité, longueur et valeur absolue) ont toutes, en un sens, un pied dans chacun des deux camps. Nous retrouverons ce schéma dans le cinquième chapitre de l'Essai sur les notions de structure et d'existence en mathématiques, dans lequel Lautman examine une autre collection de «mixtes» mathématiques. Remarquons cependant que les couples de notions dans cette liste ne sont pas des couples d'opposés conceptuels. Lautman a ceci de commun avec le philosophe éléate: nager n'est pas l'opposé de marcher, ni poisson l'opposé d'oiseau. Le fait que ces couples ne soient pas des opposés conceptuels soulève la question de savoir pourquoi le philosophe éléate divise les catégories en doublets, avec tout l'inconvénient et l'arbitraire qui en résultent, plutôt qu'en triplets, qu'en quadruplets, etc. Dans un autre ouvrage tardif mentionné par Lautman, le Timée, Platon divise les créatures vivantes en quatre classes selon l'habitat: les dieux dans les cieux, les oiseaux dans les airs, les animaux terrestres et les animaux aquatiques (39-40). De même Socrate, décrivant dans le Philèbe la méthode de division, exige seulement qu'une catégorie soit divisée en un nombre fini de sous-catégories (16D). La conception selon laquelle la dialectique relie les notions par couples est en effet présente dans le Sophiste, mais seulement sous la figure du philosophe générique venu d'Élée. Cela ne semble pas avoir été la doctrine de Platon.

La référence finale au Sophiste est plutôt indirecte. Lautman termine l'Essai sur les notions de structure et d'existence en mathématiques en consacrant quelques lignes à la conception selon laquelle il existerait un ordre développemental au sein des Idées dialectiques, et entre les Idées et les mathématiques. Il met ici à contribution les travaux d'Oscar Becker et de Julius Stenzel sur les nombres chez Platon et Aristote. Il introduit le diagramme suivant, qu'il emprunte à Stenzel.

Les itérations des Idées de l'«Un» et de la «Dyade» produisent les «Idées-nombres» (représentées par les diagrammes en arbres), qui engendrent à leur tour les nombres arithmétiques (représentés par les points noirs). La démonstration de Lautman est confuse et peu concluante. Il reproduit ce 
diagramme dans le texte principal et dans les notes en bas de page, et mentionne certaines réserves de Becker sans les discuter (p. 230, fig. 9). De toute façon, comme le note Lautman, Becker et Stenzel lisaient tous deux Platon à travers Aristote (comme le faisait également Heidegger) ${ }^{8}$. Cet exposé terminé, Lautman se tourne vers l'examen des relations entre les mathématiques et la physique. La discussion, brève et peu concluante, avec son diagramme répété sans raison, trahit chez Lautman une certaine hâte en même temps qu'une certaine insatisfaction.

\section{Le Philèbe}

Lautman mentionne une fois le Philèbe, dans le passage cité ci-dessus, en tant que source de la méthode de division. Dans ce dialogue, Philèbe, un des jeunes compagnons de Socrate, soutient que le bien pour l'homme est le plaisir. Socrate entreprend de contester cette thèse, et de soutenir que l'intelligence (incluant la connaissance et le jugement) vaut mieux que le plaisir. Avant d'exposer son argument, Socrate fait une digression méthodologique. Il décrit [5] la méthode de division (16C-17A), et affirme que la compréhension scientifique d'un sujet requiert la connaissance de la structure générée par distinctions successives. Contrairement au philosophe éléate, Socrate admet qu'une catégorie puisse se diviser en plus de deux sous-classes; il exige seulement que le nombre de sous-classes soit fini (16D).

Au début du dialogue, Socrate fait remarquer qu'une vie purement intellectuelle ne convient pas aux hommes (21E). La vie bonne pour les hommes doit inclure jusqu'à un certain degré le plaisir des sens en plus de l'activité intellectuelle - bien que, s'agissant de Platon, le côté intellectuel ait la priorité. Le point crucial est que le bien pour les hommes est un mixte d'éléments hétérogènes (sensuels et intellectuels). Cela pourrait constituer un paradoxe, si la méthode de division avait pour but d'établir une taxinomie comme chez Aristote. Dans la «logique aristotélicienne du genre et de l'espèce ", un objet semblant appartenir à deux espèces différentes constituerait pour la taxinomie un contre-exemple (comme l'ornithorynque, par exemple, représente une menace pour la catégorie «mammifère»). Par contraste, un système d'Idées platonicien est en quelque sorte antérieur aux (et indépendant des) objets qui participent à ces Idées. Un objet peut participer à plus d'une Idée (par exemple, un objet physique peut être à la fois rouge et rond). Ce mixte d'Idées se retrouve dans d'autres dialogues. Il est l'un des motifs explicatifs principaux dans la physique du Timée (34-35, 59-61), et nous l'avons déjà rencontré dans le Sophiste.

Pour ce qui nous intéresse, la conclusion significative du Philèbe est que toute vie humaine doit constituer un mixte de biens sensuels et intellectuels.

8. Heidegger (2003), p. 8. Lautman n'était pas le seul à trouver plus évocatrice que claire la lecture que Stenzel fait des écrits de Platon sur l'arithmétique. Voir Cornford (1924) et Shorey (1924). 
La nature exacte de ces biens et les liens qu'ils entretiennent les uns avec les autres varieront de vie en vie. L'amour du vin développera peut-être chez quelqu'un, sur le plan intellectuel, un savoir de connaisseur. Pour prendre un exemple différent, le travail intellectuel peut procurer du plaisir et de la satisfaction (bien que cela ne soit pas, chez Platon, la raison pour laquelle ce travail est bon). On peut imaginer une vie dans laquelle l'intelligence et le plaisir seraient des domaines entièrement séparés, quoiqu'il soit difficile d'imaginer qu'on puisse désirer une telle existence. Toute vie humaine incarnera cette dialectique d'une manière ou d'une autre, comme le révélera l'examen philosophique. Dans les vies où l'un ou l'autre de ces éléments est absent, ce manque se manifestera inévitablement sous forme d'inadéquation ou d'insatisfaction. De fait, nous aurions besoin de cette dialectique du sensuel et de l'intellectuel pour comprendre les sautes d'humeur et les changements à l'intérieur d'une vie particulière. La structure dialectique d'arrière-plan est ce qui permet d'expliquer pourquoi une vie adonnée de manière excessive, soit aux plaisirs sensuels, soit aux biens intellectuels, serait insatisfaisante.

Le Philèbe nous fournit par conséquent une analogie éthique de la caractérisation lautmanienne des Idées dialectiques en mathématiques. Dans la terminologie de Lautman, le plaisir et l'intelligence sont des «notions », et la possibilité de relations entre elles est une "Idée». Cette structure dialectique ne spécifie pas quels plaisirs et quelles pensées verront effectivement le jour. Comme Lautman le dit à propos des Idées mathématiques: "étant seulement dessin de positions éventuelles, elles n'entraînent pas forcément l'existence d'êtres particuliers susceptibles de soutenir entre eux les relations qu'elles ébauchent» (p. 243). Ces notions entrent en relation à travers l'interaction de pensées et de plaisirs particuliers, et il est impossible de prédire les détails de cette interaction à partir de la seule structure dialectique. Le savoir du connaisseur en vins influe sur son plaisir à boire. Le plaisir et l'intelligence se combinent de manière fort différente (mais non moins intimement) au cours des rares mais [6] précieux moments d'intuition dans le travail d'un scientifique. De la même façon, Lautman soutient qu'il y a une variété infinie de manières dont une relation dialectique entre notions peut se manifester dans les mathématiques réelles, et ajoute que ce n'est pas l'affaire des philosophes de tenter de prédire ou de circonscrire ces relations (p. 229).

\section{Le Timée}

Les deux références de Lautman au Timée (pp. 231, 267) nous rappellent l'une et l'autre que, selon Platon, la création d'un monde matériel est possible seulement si le "réceptacle d'une qualification géométrique» (p. 231), désigné par le terme de «lieu» (p. 267), existe déjà̀ . Il est important de spécifier que des objets différents peuvent (à des temps différents) occuper le 


\section{2 • Philosophiques / Printemps 2010}

même lieu. Le «lieu» dépend ainsi pour son intelligibilité d'un couple dialectique antérieur à lui, celui du même et de l'autre. Comme nous l'avons vu précédemment, Lautman considère la "distinction [...] entre les propriétés intrinsèques [d'un objet] et ses possibilités d'action" sur d'autres objets comme une expression de la relation du même et de l'autre. Dans les deux cas, la référence au Timée permet à Lautman de passer de la philosophie des mathématiques à la philosophie de la physique. Lautman soutient que le monde naturel est mathématiquement intelligible parce que les mêmes structures dialectiques sous-tendent tant la physique que les mathématiques. Il mentionne pour illustrer son propos les cristaux énantiomorphiques, qui constituent selon lui un exemple d'un phénomène physique dans lequel les opposés dialectiques (ici, la symétrie et la dissymétrie) sont "mélangés ". (Le présent article ne discutera pas plus avant la philosophie lautmanienne de la physique). Comme dans son allusion aux travaux de Stenzel sur les nombres, Lautman tente ici d'illustrer sa pensée selon laquelle l'intelligibilité des mathématiques et de la physique requiert un ordre dialectique antérieur. Sa démonstration achoppe dans les deux cas, du fait de l'incapacité de Lautman à expliquer ce que "priorité dialectique " veut dire.

Selon Lautman, par conséquent, la méthode de division révèle des «notions" dialectiques (dans l'acception spéciale que Lautman donne à ce terme), et avec elles les Idées des relations entre ces notions. Toutefois, Lautman ne propose pas des séries de distinctions. Ses notions ne se présentent pas sous formes d'arbres comme celles du philosophe dans le Sophiste. Comme le suggère la citation au début de cette section, ce qu'il emprunte aux dialogues tardifs est l'idée selon laquelle un particulier peut participer à des catégories hétérogènes de manière simultanée. Dans certains des exemples qu'il donne, les notions «mixées » dans telle théorie mathématique sont simplement différentes (telles que la notion d'ordinal et celle de fermeture), tandis que dans d'autres elles sont opposées (comme les cas dans lesquels il voit des mixtes de mathématiques finies et infinies).

\section{Platon ne suffit pas}

Lautman a parsemé ses œuvres de références à Platon; Heidegger, par contre, n'apparaît nulle part dans ses textes, excepté au cours de la discussion dans les «Nouvelles recherches sur la structure dialectique des mathématiques» et de manière implicite dans quelques brèves remarques de la conclusion de l'Essai sur les notions de structure et d'existence en mathématique ${ }^{10}$. Nous pouvons par conséquent supposer que Lautman s'est tourné vers Heidegger

10. Pp. 228-229. La seule exception se trouve dans un court article datant de 1933 et intitulé: "Considérations sur la logique mathématique». Mais ici Lautman discute de l'usage que les intuitionnistes ont fait de la phénoménologie et n'y exprime pas ses vues personnelles: "Les intuitionnistes se rattachent par là aux phénoménologues disciples de Husserl, Heidegger et Oscar Becker », p. 43. 
afin de résoudre un problème particulier dans la conception qu'il se faisait [7] du platonisme en général ${ }^{11}$. En outre, le texte heideggerien auquel il se réfère, Sur l'essence du fondement (Vom Wesen des Grundes), constitue une méditation sur la "différence ontologique " entre les concepts "ontiques" employés dans les sciences et les concepts «ontologiques» sous-jacents révélés par la phénoménologie. La relation entre la dialectique et les mathématiques était manifestement problématique pour Lautman. D'un côté, il est demeuré fidèle à sa conception platoniste selon laquelle les Idées sont d'une manière ou d'une autre antérieures à la matière qu'elles dominent, et qui participe d'elles. Dans une conférence prononcée en 1937, Lautman affirme que "la réalité inhérente aux théories mathématiques leur vient de ce qu'elles participent d'une réalité idéale qui est dominatrice par rapport à la mathématique, mais qui n'est connaissable qu'à travers elle» (pp. 67-68). Il savait que les tenants de l'empirisme logique (le courant dominant à son époque) considéreraient ses vues comme une mystification, "aussi obscures que les croyances mystiques à la participation du sujet à l'objet chez les primitifs dont parle M. Lévy-Bruhl» (p. 64). Lautman rétorque qu'au contraire, l'empirisme (qu'il soit aristotélicien ou viennois) coupe la pensée de l'expérience et rend par conséquent mystérieux le fait que la nature soit pour nous mathématiquement intelligible. Qui plus est, une conception tautologique des mathématiques coupe la découverte de la vérité de la quête de la vérité (puisque les tautologies ne requièrent aucune référence à une réalité quelle qu'elle soit). L'empirisme, selon lui, prive la science de sa dignité spirituelle et de sa valeur. Il est par conséquent vital, tant sur le plan scientifique que sur le plan spirituel, d'insister sur la réalité des notions dialectiques et sur les Idées de leurs relations possibles antérieurement à leur réalisation dans des cas particuliers. D'un autre côté, les notions entrent en relation les unes avec les autres seulement quand elles se trouvent «mixées " dans des particuliers. Vers la fin de l'Essai sur les notions de structure et d'existence en mathématique, Lautman décrit les Idées de relations possibles entre notions comme des «problèmes » ou des "questions », et les relations réelles (réalisées) entre notions comme des "schémas logiques":

Les schémas logiques que nous avons décrits ne sont pas antérieurs à leur réalisation au sein d'une théorie; il manque en effet à ce que nous appelons plus haut l'intuition extra-mathématique de l'urgence d'un problème logique une matière à dominer pour que l'idée de relations possibles donne naissance au schéma de relations véritables (p. 229).

11. Ce qui ne veut pas dire que Lautman ait choisi Heidegger de manière arbitraire, étant donné les références de ce dernier à Platon; Heidegger a placé au tout début d'Être et temps une citation tirée du Sophiste (244a), et il a consacré ses conférences de 1924-1925 à ce même dialogue. 


\section{4 • Philosophiques / Printemps 2010}

Avant le développement de la théorie mathématique qui résout le problème, seule préexiste "l'expérience de [l'] urgence des problèmes" $(\mathrm{p} .229)^{12}$. Une telle formulation donne cependant à entendre que c'est la psychologie des mathématiciens qui nous importe. Ce n'est pas ce que Lautman avait à l'esprit. Dans l'introduction à l'Essai sur les notions de structure, il conclut une discussion sur Hilbert et Brunschvicg en affirmant qu' "entre la psychologie du mathématicien et [8] la déduction logique, il doit y [...] avoir place pour une caractérisation intrinsèque du réel» (p. 129).

Lautman a par conséquent besoin d'un idiome philosophique dans lequel ce sentiment de l'urgence d'un problème est plus qu'un simple sentiment. Car si ce sentiment d'urgence n'est rien de plus qu'un besoin psychologique, alors son contenu ne peut avoir la signification logique qu'exige le platonisme lautmanien. En même temps, cette «intuition extra-mathématique de l'urgence d'un problème logique» ne peut être une mystérieuse sensibilité à un monde des Idées qui existerait antérieurement à l'activité des mathématiciens. Nous avons déjà vu qu'il rejette cette sorte de platonisme naï. C'est afin de combler ce besoin que Lautman se tourne vers Heidegger.

\section{Heidegger'3}

Lautman était familier avec Être et temps (voir sa référence à cet ouvrage p. 240), mais il fait appel à un ouvrage de Heidegger beaucoup plus court, intitulé Sur l'essence du fondement (Vom Wesen des Grundes) (1928). Heidegger s'efforce dans ce traité de clarifier sa distinction entre "ontologique» et «ontique» (ou, pour le dire autrement, entre «être» et «êtres»). Il développe à cet effet une méditation sur l'histoire de la philosophie défiant le résumé, mais dont l'idée centrale est la suivante: Kant nous a appris que la métaphysique (c'est-à-dire, dans la terminologie heideggerienne, l'" ontologie ") ne s'intéresse pas directement à ce qu'est le monde "en lui-même ". L'ontologie révèle plutôt au premier chef la structure profonde de la manière dont nous appréhendons le monde et nous nous y comportons. Dans cet exposé, "le monde» devrait être compris dans le sens qu'il a quand on dit que les gens adonnés à la magie sympathique «vivent dans un monde différent» de ceux habitués dans la poursuite de leurs objectifs, à user des méthodes scientifiques modernes. En ce sens, seuls les humains vivent dans «le monde». Les chats et les chiens occupent un lieu physique, mais ils ne possèdent pas un sens profond et en grande partie inarticulé de ce qu'est le monde et son fonctionnement pour leur servir à déterminer et à orienter leurs actes. C'est ce trait crucial chez les humains qui nous intéresse. Nos questions peuvent être pratiques (telles que: «Pourquoi est-ce que je ressens

12. «Le seul élément a priori que nous concevions est donné dans l'expérience de cette urgence des problèmes..." , p. 229.

13. Je suis reconnaissant au docteur Nicholas Joll pour son aide généreuse dans la lecture de Heidegger. 
cet élancement dans le genou ?» ou «Me faudra-t-il mélanger plus de ciment pour finir ce mur ?") ou elles peuvent être le fait d'une science avancée. Scientifique ou non, chaque question comporte certaines hypothèses quant à la forme de la réponse. Un dormeur éveillé par un bruit pourra demander: «Qu'est-ce que c'est ?» ou "Qui est là ?», ou «Est-ce que j’ai imaginé cela ?", suivant ses attentes et son état d'esprit. Cela est vrai même des questions qui semblent ne faire aucune hypothèse, comme: "Pourquoi y a-t-il quelque chose plutôt que rien ? ». Nous n'avions pas à poser la question à propos des choses. Nous aurions pu demander: "Pourquoi y a-t-il de la matière plutôt que du vide ? ". Les disciplines scientifiques ont leurs manières caractéristiques d'appréhender le monde: le physicien moderne pose ses questions dans le langage des mathématiques. Les premiers étudiants de la nature, qui "vivaient dans un autre monde" au sens phénoménologique, auraient trouvé cette manière de faire inintelligible (même s'ils avaient compris les mathématiques). Ainsi, nos questions révèlent toujours quelque chose des traits structuraux profonds du monde tel que nous l'appréhendons.

[9] Qu'en est-il dès lors de la «différence ontologique » ? Dans un passage cité par Lautman, Heidegger déclare:

La détermination anticipée de l'Être d'une nature en général (essence et modalité) prend corps dans les "concepts fondamentaux" de la science correspondante. Ces concepts délimitent par exemple, ce que sont l'espace, le lieu, le temps, le mouvement, la masse, la force, la vitesse. [...] [Ces concepts scientifiques concernent l'existant, et non l'être, ils ne] recèlent point en eux les authentiques concepts ontologiques de l'être de l'existant qui est mis en question par cette science ${ }^{14}$.

En d'autres termes, même les concepts les plus fondamentaux utilisés par les scientifiques sont simplement ontiques. Les concepts ontologiques correspondants se situent hors des ressources conceptuelles de la science. Lautman soutient que la même chose est vraie des notions et des Idées dialectiques qu'il discerne à l'œuvre dans les mathématiques. «La dialectique, affirme-t-il, ne fait pas partie des mathématiques, et ses notions sont sans rapport avec les notions primitives d'une théorie » (p. 242). Bien au contraire, la dialectique est ontologiquement antérieure aux mathématiques, au sens heideggerien d' "ontologique ». Dans la conclusion de l'Essai sur les notions de structure et d'existence en mathématique, Lautman décrit sa philosophie des mathématiques comme une enquête phénoménologique sur l'intuition extra-mathématique ou le souci de l'«urgence» d'un problème logique.

14. Martin Heidegger, «Sur l'essence du fondement » ( Vom Wesen des Grundes»), in Qu'est-ce que la métaphysique? traduit par Henry Corbin, Paris, Gallimard, 1938, pp. 57-58, et tel que cité par Lautman, p. 241. 
«Souci » doit être compris ici comme sorge (préoccupation ou soin), au sens heideggerien du terme.

La phénoménologie heideggerienne s'intéresse essentiellement à nos habitudes mentales et à nos attentes, telles que révélées dans nos questions (ou plutôt, dans nos pratiques d'enquête). Toutefois, cette enquête sur la structure de notre subjectivité active et questionnante révèle également l'ordre de la réalité objective. Heidegger emprunte à Kant l'idée que l'ordre cohérent de la subjectivité humaine et l'ordre cohérent du monde sont deux côtés d'un même fait. À un niveau de profondeur inexprimable, d'une manière ou d'une autre, trois aspects se rejoignent: la structure de notre subjectivité, l'activité intense que nous déployons dans l'application de choses à d'autres choses, et les structures profondes que nous découvrons au sein du monde.

L'explication heideggerienne que propose Lautman de l'objectivité des mathématiques semble se ramener à ceci: les théories mathématiques "participent » des Idées dialectiques, dans ce sens qu'elles relient entre elles des notions dialectiques. Les Idées posent de vagues et nébuleuses questions (suggérant, ce faisant, la possibilité de relations notionnelles) auxquelles les mathématiques fournissent des réponses précises et détaillées. En ce sens, les Idées appellent les théories mathématiques à l'existence (quoique nous ne devrions pas nous attendre à discerner les Idées à l'origine de l'appel avant que la théorie mathématique ne soit complète). Les théories mathématiques dépendent par conséquent des Idées pour leur objectivité. Les Idées sont objectives en ce sens qu'elles font partie de la structure profonde de notre engagement avec le monde, ce qui signifie qu'elles font partie de notre propre structure profonde, ou, ce qui revient au même, qu'elles font partie de la structure profonde de notre monde. Puisque notre monde est le seul que nous connaissions, nous pouvons aussi bien dire qu'elles font partie de la [10] structure profonde du monde, tant et aussi longtemps que nous nous souvenons que cette manière de voir est ontologique, et non pas ontique. Elle relève de la phénoménologie, pas de la science.

\section{Les exemples mathématiques de Lautman}

Ayant esquissé de façon abstraite les vues de Lautman, je considérerai à présent les exemples mathématiques qu'il fournit. La première des deux thèses de Lautman (Essai sur l'unité des sciences mathématiques dans leur développement actuel) prend comme point de départ une distinction faite par Hermann Weyl dans son ouvrage de 1928 sur la théorie des groupes et la mécanique quantique. Weyl trace une distinction entre la mathématique «classique ${ }^{15}$, qui a trouvé son plus haut épanouissement dans la théorie des fonctions de variables complexes, et la mathématique «moderne» (ou

15. N.B. Lautman utilise dans ce passage les expressions «la mathématique classique » et «la mathématique moderne», au singulier (NDLT). 
nouvelle) représentée (par exemple) par la théorie des groupes et les algèbres abstraites, la théorie des ensembles et la topologie ${ }^{16}$. Pour Lautman, la mathématique "classique» dans la distinction weylienne correspond pour l'essentiel à l'analyse, c'est-à-dire aux mathématiques qui dépendent d'une variable quelconque tendant vers zéro: convergence des séries, passage à la limite, continuité, dérivation et intégration. Ce sont les mathématiques des voisinages arbitrairement petits, et elles ont atteint leur plein développement au dix-neuvième siècle. Par contre, la mathématique «moderne» dans la distinction weylienne est "globale»; elle étudie les structures des " touts ${ }^{17}$. La topologie algébrique, par exemple, considère les propriétés d'une surface entière (combien de trous ?) plutôt que les agrégations de voisinages. Après avoir cité et illustré par des exemples la distinction weylienne, Lautman la reformule comme suit:

[...] en opposition à l'analyse des continus et de l'infini, les structures algébriques ont un aspect nettement fini et discontinu. Quelle que soit l'infinité des éléments qui constituent un groupe, un corps, une algèbre (au sens restreint du mot), les méthodes de l'algèbre moderne consistent le plus souvent à imposer à ces éléments une division en classes d'éléments équivalents, et à substituer ainsi à un ensemble infini la considération d'un nombre de classes qui, dans les applications, est le plus souvent fini (op. cit., p. 86-87).

L'essentiel de la thèse lautmanienne sur l' 'unité» est constitué de quatre exemples ${ }^{18}$ dans lesquels les théories de l'analyse moderne (c'est-àdire de l'analyse pratiquée au vingtième siècle) dépendent dans leurs moindres détails des résultats et des techniques empruntés à la branche algébrique «nouvelle» de la distinction weylienne. Dans ces quatre cas, l'algèbre vient à la rescousse de l'analyse. Lautman transforme ainsi une distinction historique générale (entre les mathématiques du dix-neuvième siècle, locales, analytiques, continues et infinies, et les mathématiques nouveau style, "globales ", synthétiques, discontinues et finies) en une famille de dyades dialectiques (celles du local et du global, de l'analytique et du synthétique, du continu et du discontinu, de l'infini et du fini). Ces couples ne sont pas des oppositions vides. Ils trouvent leur contenu dans les détails de théories mathématiques [11] qui, même si elles relèvent de l'analyse, empruntent parfois un point de vue caractéristique de l'algèbre. En d'autres termes, «les

16. 2006, pp. 83-84.

17. Op. cit., p. 84

18. Celui des «décompositions dimensionnelles en théorie des fonctions"; celui du "rôle des métriques non euclidiennes de la théorie des fonctions analytiques"; celui de «l'algèbre non commutative [intervenant] dans la théorie de l'équivalence des équations différentielles"; enfin, celui de l'usage de "structures algébriques finies et discontinues pouvant servir à déterminer l'existence de fonctions de variable continue », p. 87. 
méthodes sont algébriques mais les résultats s'étendent à l'analyse ${ }^{19}$. Parvenu à ce point, nous avons laissé le dix-neuvième siècle derrière nous, et nous nous intéressons aux «mixtes» analytiques et algébriques dans les mathématiques contemporaines (du vingtième siècle).

Dans son autre grande thèse, Essai sur les notions de structure et d'existence en mathématiques, Lautman donne à sa pensée dialectique une expression plus philosophique et plus polémique. Cette seconde thèse est composée de six chapitres, les trois premiers étant consacrés aux «schémas de structure» et les trois derniers aux "schémas de genèse». Les trois schémas de structure sont: «le local et le global ", "les propriétés intrinsèques et les propriétés induites » et «la montée vers l'absolu » (malencontreusement dénommée $)^{20}$. Les deux premiers de ces trois schémas sont des couples semblables à ceux déjà rencontrés dans la thèse de Lautman sur l'«unité». La «montée vers l'absolu» appartient à un genre différent; elle implique un progrès, depuis les objets mathématiques qui sont en un certain sens «imparfaits», vers un objet qui est «parfait» ou «absolu». Lautman en donne deux exemples mathématiques: la théorie du corps de classes, qui "monte» vers le corps de classes absolu, et les surfaces de recouvrement d'une surface donnée, qui «montent » vers une surface universelle de recouvrement simplement connexe. Dans chacun des cas, on retrouve une séquence correspondante de sous-groupes imbriqués les uns dans les autres (y compris le sous-groupe trivial correspondant au corps de classes «absolu» ou à la surface «absolue»), qui induit une structure en «escalier» dans la «montée ». (Lautman introduit cette idée par le biais d'une brève discussion sur la correspondance de Galois, pp. 166-168.) Ce schéma dialectique diffère en partie des autres. Les exemples cités antérieurement consistaient en des couples de notions (fini/infini, local/global, etc), dans lesquels aucun membre d'aucun couple n'était inférieur à l'autre ${ }^{21}$. Comme nous l'avons vu précédemment, Lautman soutient que, dans certaines occasions, les mathématiques finies permettent d'appréhender les mathématiques infinies (pensons par exemple à l'usage des corps d'entiers finis dans l'étude des nombres naturels infinis dénombrables). En mathématiques, le fini n'est pas une version en quelque sorte imparfaite de l'infini. De la même façon, les mathématiques «locales» de l'analyse peuvent dépendre pour leurs fondations de la topologie "globale» (comme le soutient Lautman, p. 85), mais les premières ne sont pas une version bâclée ou en quelque façon inadéquate des dernières. Lautman introduit la section sur la "montée vers l'absolu» en rappelant

19. «[...] il est possible de retrouver dans les théories modernes de l'analyse les points de vue qui caractérisent l'algèbre[,] [...] théories [...] dont les méthodes sont algébriques, et dont les résultats s'étendent à l'analyse ", p. 121.

20. La résonance hégélienne de «l'absolu» est un leurre; il semble que Bernays se soit laissé duper (Bernays (1940), p. 20).

21. Mais voir aussi Barot (2003), p. 12. Rappelons également que, pour Platon, l'intellectuel est supérieur au sensuel. 
l'argument de Descartes, dans lequel celui-ci raconte la manière dont ses propres imperfections l'ont conduit à reconnaître l'existence d'un être parfait (Dieu). L'homme (pour Descartes) n'est pas l'opposé dialectique de Dieu, ni n'en constitue une alternative; l'homme est plutôt une image imparfaite de son créateur. Dans un mouvement de pensée similaire, selon Lautman, la réflexion sur les corps de classes et sur les surfaces de recouvrement «imparfaits» conduit les mathématiciens, respectivement, aux corps de classes et aux surfaces de recouvrement «parfaits» et «absolus». Bref, la «montée vers l'absolu» introduit une structure dialectique différente des couples de notions rencontrés jusqu'à présent. Cette structure n'a rien en commun avec les motifs découverts dans les trois dialogues platoniciens.

Les trois schémas de genèse sont présentés successivement dans trois chapitres intitulés : "Essence et existence », "Les mixtes » et "Du caractère exceptionnel de l'existence». Dans les deux premiers de ces chapitres, la structure d'un domaine mathématique engendre des objets mathématiques nouveaux; dans le troisième chapitre, [12] Lautman considère des cas dans lesquels un objet est redevable pour son existence à des propriétés exceptionnelles qui le distinguent d'un ensemble établi d'objets. Lautman n'aborde pas directement la question générale du statut métaphysique des objets mathématiques. Il s'intéresse plutôt à la manière dont les structures et les objets mathématiques sont générés à partir des mathématiques déjà existantes. Comme il l'explique lui-même, les rôles de «structure d'origine "22 et d' "objet créé ${ }^{23}$ sont relatifs; les objets qui doivent leur existence à la structure d'un autre domaine peuvent à leur tour servir de structure d'origine («de domaine de base») pour la genèse d'une nouvelle classe d'entités (p. 187). Lautman vise en partie à s'opposer à la conception selon laquelle l'existence mathématique se confondrait purement et simplement avec la consistance d'un système axiomatique. Lautman s'est attardé sur les difficultés techniques communément engendrées par les tentatives pour prouver la consistance d'un système (pp. 179-187). Mais son véritable argument est que les entités mathématiques ne dépendent pas pour leur existence de décisions apparemment arbitraires conduisant à considérer certains ensembles d'axiomes au détriment d'autres. Les mathématiciens créent plutôt de nouvelles structures mathématiques en répondant aux questions latentes dans l'ordre dialectique extra-mathématique sous-jacent. Ici aussi, les structures dialectiques repérées par Lautman sont plus richement variées que ses références à Platon ne pourraient le suggérer.

\section{Brouiller la différence ontologique}

Lautman conclut l'Essai sur les notions de structure et d'existence en mathématiques avec quelques remarques qui, bien que le nom de Heidegger n'y

22. Lautman utilise l'expression «domaine de base», p. 187 (NDLT).

23. Lautman utilise l'expression «être créé», p. 187 (NDLT). 
soit pas mentionné, sont clairement en lien avec la discussion faisant explicitement référence à Heidegger dans les Nouvelles recherches (pp. 228-229). Après une brève discussion sur Platon (pp. 230-234), Lautman termine sa thèse avec l'énoncé de son credo, qu'il tient pour vrai tant des mathématiques que de la physique:

La nature du réel, sa structure et les conditions de sa genèse ne sont connaissables qu'en remontant aux Idées dont la science incarne les liaisons ( p. 234).

Comme nous l'avons vu précédemment, Lautman fait appel à Heidegger pour expliquer la relation entre la dialectique et les mathématiques. Tout le but de Sur l'essence $d u$ fondement est d'insister sur la différence ontologique, c'est-à-dire sur la distinction entre l'ontologique et l'ontique. La division du travail entre le scientifique et le philosophe dépend de cette distinction. Le scientifique utilise des concepts ontiques pour établir des vérités ontiques; le philosophe en révèle l'ontologie correspondante. Lautman insiste sur la distinction entre la dialectique et les mathématiques. Si la dialectique tentait de trouver des solutions qui lui soient propres aux problèmes qu'elle exprime, elle «imiterait à ce point les mathématiques avec tout un ensemble de distinctions subtiles et d'artifices de raisonnement qu'elle se confondrait avec les mathématiques elles-mêmes ${ }^{24}{ }^{2}$. C'est, laisse entendre Lautman, le sort qui attend le logicisme de Frege et de Russell. Les notions et les idées dialectiques doivent pouvoir s'exprimer dans des exemples mathématiques. L'expression dans des exemples mathématiques soumet une Idée à [13] « tout un cortège de précisions, de limitations et d'exceptions où s'affirment et se construisent les théories mathématiques ${ }^{25}$ ». Ainsi, nous pourrions, par exemple, examiner les différents concepts mathématiques de complétude et de fermeture, et reconnaître en eux des versions mathématiques de la notion vague (et donc présumément dialectique) selon laquelle un élément complexe peut être auto-suffisant ou sui generis. Pour rappeler l'un des exemples détaillés fournis par Lautman, celui-ci nous invite à voir les relations mathématiques entre les propriétés intrinsèques et relationnelles des objets mathématiques comme une spécification mathématique de la dialectique du même et de l'autre.

Mais nous avons maintenant un problème. Comment parvenir à distinguer entre l'activité légitime consistant à chercher des réponses mathématiques aux questions dialectiques, et l'activité illégitime consistant à faire de la dialectique une imitation des mathématiques ? Après tout, historiquement, les

24. «Une dialectique qui s'engagerait dans la détermination des solutions que ces problèmes logiques peuvent comporter se verrait entrainnée à constituer tout un ensemble de distinctions subtiles et d'artifices de raisonnement qui imiteraient à ce point les mathématiques qu'elle se confondrait avec les mathématiques elles-mêmes", p. 228.

25. «Il faut ensuite, pour que l'exemple supporte l'Idée, apporter à celle-ci tout un cortège de précisions, de limitations et d'exceptions où s'affirment et se construisent les théories mathématiques », p. 243. 
mathématiques n'ont pas de frontières fixes. Par exemple, Euler pensait que le problème des ponts de Königsberg se situait en dehors des mathématiques, parce que «la solution [en était] fondée sur la seule raison, et sa découverte ne dépend[ait] d'aucun principe mathématique ${ }^{26} »$. La logique formelle est demeurée en dehors des mathématiques pendant plus de deux mille ans (c'està-dire pendant la période allant des Premiers Analytiques d'Aristote à la publication de l'Analyse mathématique de la logique de Boole en 1847). La distinction lautmanienne entre dialectique et mathématiques dépend non seulement de l'autorité de Heidegger, mais encore de l' 'insuffisance essentielle» (p. 243) des Idées dialectiques, c'est-à-dire du fait qu'elles ne peuvent pas être comprises, excepté à travers le développement de la théorie mathématique. Toutefois, cela est également vrai des concepts mathématiques primitifs ou non développés. Les concepts primitifs du continu et de l'infini ont soulevé des questions auxquelles furent apportées des réponses appropriées seulement grâce à l'étude d'exemples mathématiques et l'articulation des théories mathématiques. Tout cela fait que le continu et l'infini sont clairement des concepts mathématiques, aussi primitifs soient-ils.

Les exemples fournis par Lautman lui-même suggèrent que la ligne de démarcation entre la dialectique et les mathématiques n'est ni claire ni stable. Considérons encore une fois le diagramme emprunté à Stenzel. Les treillis n'étaient pas des objets mathématiques à l'époque de Platon, mais ils le sont aujourd'hui. Devons-nous supposer que la structure dialectique sousjacente de l'arithmétique platonicienne constitue elle-même un exemple d'un concept mathématique (à savoir, un treillis), présumément doté d'une base dialectique qui lui soit propre ? Lautman rejette explicitement les régressions de ce type (p. 232).

La deuxième partie des «Nouvelles recherches sur la structure dialectique des mathématiques" consiste en l'examen de deux cas qui font ressortir le contraste entre l'analyse et l'algèbre rencontré une première fois dans l'Essai sur l'unité des sciences mathématiques. Dans ce premier essai, nous avons vu l'algèbre venir en aide à l'analyse. Dans les deux cas dont il est question ici, nous voyons l'analyse (les mathématiques du continu) fournir des preuves à la théorie des nombres. Le deuxième cas constitue une assez bonne confirmation de l'argument de Lautman en faveur de l'unité des mathématiques: il consiste à utiliser la fonction zêta de Riemann pour l'investigation de la densité des nombres premiers. Le premier cas est quelque peu artificiel: il s'agit de la preuve de Hecke de la réciprocité quadratique. Il est artificiel parce que (comme le reconnaît Lautman) il n'est nul besoin de faire appel à l'analyse pour prouver ce théorème. À propos de la preuve de Hecke de la réciprocité quadratique, Lautman écrit : [14]

26. Euler, Opera Omnia (4), I, lettre 590 (NDLR: ma traduction). Euler s'est demandé si c'était cela qu'entendait Leibniz par «géométrie de la position». 
[...] l'outil analytique, c'est-à-dire les fonctions, sert à démontrer un résultat arithmétique, parce que la structure de l'outil et celle du résultat participent l'une et l'autre d'une même structure dialectique, celle que pose le problème de la réciprocité de rôles entre éléments inverses l'un de l'autre (p. 248).

Cette remarque soulève deux problèmes pour la distinction entre la dialectique et les mathématiques. Premièrement, la réciprocité est une sorte de symétrie. La symétrie qui se manifeste entre ces éléments mutuellement inverses ne requiert sans doute pas l'appareil de la théorie des groupes, mais elle constitue néanmoins un concept mathématique, tout comme les relata d'ailleurs. Deuxièmement, la relation appropriée entre la dialectique et les mathématiques semble avoir été inversée. Dans une lettre adressée au mathématicien Maurice Fréchet, Lautman explique que:

[...] c'est dans la mesure où une théorie mathématique apporte une réponse à un problème dialectique définissable mais non résoluble indépendamment des mathématiques que la théorie me paraît participer, au sens de Platon, à l'Idée vis-à-vis de laquelle elle est dans la même situation que la Réponse par rapport à la Question, [...] (p. 260).

En principe, par conséquent, la dialectique est à l'égard des mathématiques ce qu'une question est à l'égard d'une réponse, sauf qu'ici une question mathématique (pourquoi tel corpus de la théorie analytique sert-il à prouver tel résultat arithmétique ?) reçoit une réponse dialectique. En tout cas, "faire partie de la même structure dialectique que " est une relation symétrique, mais non pas la relation entre outil et résultat. Lautman donne d'autres exemples dans les "Nouvelles recherches» dans lesquelles (soutient-il) «la convergence des théories mathématiques différentes résulte de leur affinité de structure dialectique»(p. 250), mais il donne ailleurs des exemples de théories mathématiques qui partagent les mêmes structures dialectiques (telles que tous les exemples concernant la dyade même/autre) et qui ne montrent cependant aucun signe de convergence.

Bref, l'affirmation selon laquelle la science et la phénoménologie traitent de différents concepts s'effondre dans la pratique mathématique ${ }^{27}$. La symétrie (par exemple) est un concept mathématique, mais elle peut également fonctionner comme une notion dialectique au sens de Lautman — c'est l'une des notions qui donnent forme à nos questions. Si elle le fait explicitement, elle peut également fonctionner de manière heuristique au sens que Pólya donne à ce terme. Lautman ne parle pas d'heuristique; au contraire, il affirme que nous ne devons pas nous attendre à discerner une question dialectique avant d'arriver à sa réponse mathématique. Une telle affirmation néglige cependant le fait (sur lequel Lautman insiste dans d'autres écrits) que les mêmes notions et Idées dialectiques peuvent figurer dans différentes théories

27. Pour un argument indépendant avec une conclusion similaire, voir Barot (2003), pp. 14-17. 
mathématiques. Au fur et à mesure qu'une notion ou Idée reparaît dans diverses théories mathématiques, elle peut devenir une partie explicite de la culture mathématique et se mettre par conséquent à fonctionner heuristiquement. Reconnaitre qu'un concept puisse fonctionner de part et d'autre de la distinction entre dialectique et mathématiques s'accorderait [15] avec ce que nous retrouvons dans le Platon tardif. La dyade "même/autre " peut jouer un important rôle ontologique, en conférant une intelligibilité à des concepts aussi divers que «lieu » et «intrinsèque/extrinsèque », mais elle remplit également un rôle dans des questions empiriques banales telles que: "Est-ce le même chien que j'ai vu hier ?". Par conséquent, si nous souhaitons que Lautman enrichisse notre propre philosophie, la première chose à faire serait d'abandonner la "distinction ontologique". Cette vue peut même trouver un certain soutien chez Heidegger. Dans une conférence de 1936 intitulée «La science moderne, la métaphysique et les mathématiques », celui-ci écrit:

La grandeur et la supériorité de la science de la nature aux seizième et dix-septième siècles réside en ceci que tous les chercheurs d'alors étaient philosophes. Ils comprenaient qu'il n'y a pas de pur fait, mais qu'un fait n'est ce qu'il est qu'à la lumière du concept qui le fonde [...] [L]es têtes actuelles de la physique atomique, Niels Bohr and Heisenberg, pensent d'un bout à l'autre en philosophes.... ${ }^{28}$

Il est difficile de voir quel bénéfice la philosophie pourrait apporter à la science si la distinction ontologique entre elles est maintenue. L'affirmation de Heidegger selon laquelle les chercheurs de valeur sont aussi philosophes suggère que ceux-ci ne respectent pas la «distinction ontologique» dans leur pratique.

Une modification de ce genre fournirait de surcroît à Lautman une réponse à la critique formulée par l'un de ses plus proches collègues. Lors d'une rencontre tenue en février 1939, Lautman a soutenu que l'objectivité des théories mathématiques dépendent de leur participation aux Idées non mathématiques qui les dominent. Jean Cavaillès, qui était également présent, a fait la remarque suivante: "Personnellement je répugne à poser une autre chose qui dominerait la pensée effective du mathématicien, je vois l'exigence dans les problèmes..." (p. 263). L'abandon de la "distinction ontologique " permettrait à Lautman de répliquer que les Idées dialectiques dominent bel et bien les théories mathématiques, mais que les Idées, les théories et la domination font toutes partie de la pensée mathématique effective. Par conséquent, nonobstant toutes les considérations touchant la question de la «domination ", aucune contrainte étrangère ne gêne la pensée des mathématiciens ${ }^{29}$.

Traduit de l'anglais par Anne-Marie Boisvert

28. Heidegger in La question de la chose, cité en partie par Jean Beaufret et Guy Basset dans De l'existentialisme à Heidegger, Vrin , 1986, p. 163. N.B. Je n'ai pas pu retrouver le passage chez Heidegger lui-même [ NDLT].

29. Je suis reconnaissant aux docteurs Corfield, Joll et Moyal-Sharrock pour leur lecture attentive des versions antérieures de cet article. 


\section{$94 \cdot$ Philosophiques / Printemps 2010}

\section{Références}

Annas, Julia (1977). «Aristotle on Substance, Accident and Plato's Forms », Phronesis, vol. 22, $\mathrm{n}^{\circ}$ 2, pp. 146-160.

Ash, Avner \& Robert Gross. Fearless Symmetry: Exposing the Hidden Patterns of Numbers, Princeton \& Oxford, Princeton University Press, 2006.

Barot, Emmanuel. «L'objectivité mathématique selon A. Lautman : entre Idées dialectiques et réalité physique ", Cahiers François Viète, 6, 2003, pp. 3-28.

Bernays, Paul. Compte rendu de Essai sur les notions de structure et d'existence en mathématiques et de Essai sur l'unité des sciences mathématiques dans leur développement actuel de Albert Lautman, The Journal of Symbolic Logic, vol. 5, $\mathrm{n}^{\circ} 1$ (mars 1940), pp. 20-22.

Black, Max. Compte rendu de La pensée mathématique de Jean Cavaillès et Albert Lautman, The Journal of Symbolic Logic, vol. 12, $\mathrm{n}^{\circ} 1$ (mars 1947), pp. 21-22.

Cornford, F. M. Compte rendu de Zahl und Gestalt bei Platon und Aristoteles de Julius Stenzel, The Classical Review, vol. 38, n 7/8, 1924, p. 209.

Heidegger, Martin. Basic Writings, London, Routledge, Kegan and Paul, 1993.

-. Pathmarks, Cambridge: University of Cambridge Press. William McNeill (dir.), 1998.

—. Plato's Sophist, Bloomington : Indiana University Press. Traduction de Richard Rojcewicz et André Schuwer, 2003.

Lautman, Albert. Les mathématiques, les idées et le réel physique, Paris, Vrin, 2006. Introduction et biographie par Jacques Lautman; introduction de Fernando Zalamea; préface à l'édition de 1977 par Jean Dieudonné.

Lemmermeyer, Franz. Reciprocity Laws from Euler to Eisenstein, Berlin, Springer, 2000.

Plato. Timaeus, London, Penguin. Traduction de H. D. P. Lee, 1965.

-. Philebus and Epinomis, London, Dawsons. Traduction de A. E. Taylor, 1972.

- The Sophist: Part II of the being of the beautiful, Chicago and London, University of Chicago Press, 1984. Traduction de Seth Bernardete.

Shorey, Paul. Compte rendu de Zahl und Gestalt bei Platon und Aristoteles de Julius Stenzel, Classical Philology, vol. 19, n 4, 1924, pp. 381-383.

Trevaskis, J. R. "Division and Its Relation to Dialectic and Ontology in Plato", Phronesis, vol. 12, no 2, 1967, pp. 118-129.

Wilson, Robin. "Euler's combinatorial mathematics ", Bulletin of the British Society for the History of Mathematics 23 (1), 2008, pp. 13-23. 